\title{
The Fundamental Role of Prophylactic Anticoagulation Therapy and the Complications of Hypercoagulability in Patients infected with Severe Acute Respiratory Syndrome Coronavirus 2 (SARS-CoV-2)
}

Haidar Alwaeli ( $\square$ halwaeli@asu.edu )

Medical Education and Simulation, Richmond University Medical Center, New York, USA

\section{Mahvesh Shabbir}

Medical Education and Simulation, Richmond University Medical Center, New York, USA

Mali Khamissi Sobi

Family Medicine, A+ Urgent Care, Atlanta, USA

Jack Demirjian

Internal Medicine, Richmond University Medical Center, Staten island, USA

Arham Ahmad

Internal Medicine, Richmond University Medical Center, Staten island, USA

\section{Serene Musallam}

Medical Education and Simulation, University of Maryland Medical Center, Baltimore, USA

\section{Research Article}

Keywords: covid-19, coronavirus disease 2019, covid-19-associated coagulopathy, stroke and covid-19, covid-19 respiratory failure, prophylactic and therapeutic anticoagulation, covid-19 pneumonia, covid-19 neurological outcomes, covid induced ards, sars-cov-2 induced ards

Posted Date: November 9th, 2020

DOI: https://doi.org/10.21203/rs.3.rs-103650/v1

License: (c) (i) This work is licensed under a Creative Commons Attribution 4.0 International License. Read Full License 


\section{Abstract}

Coronavirus disease 2019 (COVID-19) is predominately an upper respiratory infectious disease caused by the Severe Acute Respiratory Syndrome coronavirus 2 (SARS-CoV-2). Although most patients infected with COVID-19 generally present with acute respiratory distress symptoms, we are now learning that the virus can cause a multitude of disruption in physiological and pathophysiological homeostasis. Moreover, there are increasingly reported cases of thromboembolic events occurring in infected patients, resulting in widespread use of anticoagulation therapy. This case series presents seven patients who have received anticoagulation therapy over the course of the disease. Additionally, the correlation between thromboembolic complications secondary to COVID-19, prophylactic use of anticoagulation therapy and the significant pathological findings that might arise will be assessed and discussed in great details.

\section{Introduction}

Coronavirus disease 2019 (COVID-19) is a highly contagious respiratory disease that result in acute respiratory distress, multiorgan failure, in part due to the complex role of SARS-CoV-2 and its angiotensin-converting enzyme 2 (ACE2) receptor binding capacity [1]. This marked affinity of the virus has been associated with the downregulation of ACE2 enzyme and the disruption of physiological and pathophysiological homeostasis. Moreover, a subset of patients with severe COVID-19 infection have developed a list of fatal immunological syndromes known as Cytokine Storm Syndromes (CSS), hemophagocytic syndromes, hemophagocytic lymphohistiocytosis (HLH), and macrophage activation syndrome (MAS), characterized by an overwhelming systemic inflammation, hemodynamic instability, widespread multiorgan dysfunction and potentially death [2].

An increasingly recognized feature of COVID-19 infection is extensive macrovascular thrombosis and disseminated intravascular coagulation (DIC), often resulting in brain ischemia, even in young healthy patients with no prior history of inherited hypercoagulopathies [3,4]. The comorbidity of COVID-19 related complications may be explained, in part, by a direct correlation of severe inflammatory response and the disruption of the physiological coagulation cascade.

The following is a presentation of thromboembolic complications and the efficacy of anticoagulation therapy in 7 hospitalized patients with COVID-19 infection at our institutions.

\section{Materials And Methods}

Hospitals in New York reported several cases of thromboembolic strokes, multi-organ failure, venous thromboembolism, and pulmonary embolism in hospitalized patients. This study was carried out through descriptive analyses that summarized the clinical presentation, complications and outcomes of patients between April 20 and October 10, 2020. Furthermore, 7 patient cases from two centers in New York, USA were obtained. The diagnosis of COVID19 was made based on the Center for Disease Control and Prevention (CDC) case definition. Authorized assays for the detection of SARS-CoV-2 nucleic acid or antigen was performed. One method of testing was viral nucleic acid or antigen testing from the respiratory system (nasal or oral swabs, or saliva), to establish 
whether an active SARS-CoV-2 infection is present. Such methods of testing can be utilized in both symptomatic and asymptomatic patients.

\section{Results}

Patient 1.

An 88-year-old man with a past medical history (PMH) of hypertension ( $\mathrm{HTN}$ ) and asthma was admitted with an active COVID-19 infection. Initially, he experienced self-limiting gastrointestinal symptoms of nausea and diarrhea. The patient drove to a different state and began experiencing mild symptoms of dyspnea, fever, dry cough and polydipsia. He presented to our emergency department with fever, mild dyspnea, and bilateral lung opacities on chest $x$-ray. He tested positive for COVID-19 by a real-time reverse transcriptase-polymerase chain reaction (RT-PCR) assay and was subsequently admitted. Laboratory findings included normal D-dimers and lymphopenia.

Unenhanced CT image of the chest demonstrated ground-glass opacities in both upper lobes. Patient was placed on Apixaban 2.5-5mg PO BID and was monitored for any clinical changes. The patient improved and was discharged 5 days later.

Patient 2.

A 64-year-old female with a PMH of HTN, Diabetes Mellitus II (DMII) and Systemic Lupus Erythematosus (SLE) presented to the emergency department with weakness and fatigue for three days. Her husband tested positive for active COVID-19 infection and was admitted to ICU a week prior to her presenting illness. Patient denied any associated symptoms such as fever/chills, dry cough, dyspnea, anosmia, diarrhea, nausea or loss of appetite. SARS-CoV-2 RT-PCR and chest $\mathrm{x}$-ray were ordered showing positive results and patchy peripheral infiltrates in the right midlung laterally and smaller patchy peripheral infiltrates in the left midlung respectively. Patient was admitted to general floor and later transferred to intensive care unit (ICU) due to worsening respiratory failure followed by mechanical ventilation via endotracheal tube. Patient's treatment regimen consisted of Heparin Sodium 5,000 units SC Q12HR, Enoxaparin SC Q12HR, Apixaban 5mg PO BID, Hydroxychloroquine Sulfate 200mg PO Q12HR and Remdesivir 100mg IV BID. Patient was later removed from mechanical ventilation and placed on a tracheal collar. She remained in ICU for six weeks and was transferred to the institution care facility when she was stable for safe removal of the tracheal collar.

\section{Patient 3.}

A 47-year-old male with no PMH presented to the ED with worsening cough, fever and dyspnea with an oxygen saturation of $60 \%$. Ten days prior to presenting illness, patient stated he tested positive for COVID-19, soon after his son too tested positive without symptoms. He had been experiencing fever, chills, headache, myalgia, and diarrhea but no respiratory symptoms until the day of admission which was what prompted him to seek medical attention. Patient denies other associated symptoms such as vomiting, nausea, anosmia, or loss of appetite. A SARS-CoV-2 RT-PCR was performed to confirm COVID-19 status which was indeed positive. A single AP portable radiograph of the chest was performed signifying diffuse airspace consolidation involving the right lung, retrocardiac, and left base suspicious for pneumonic airspace infiltrate. Patient was placed on 100\% non-rebreather mask and transferred to ICU. He was then mechanically ventilated days later due to worsening respiratory failure and remained therein 
until stabilization. He was treated with Enoxaprin Sodium 80 mg SC Q12HR, Remdesivir 100mg/Sodium Chloride $250 \mathrm{ml} / \mathrm{hr}$ IV BID, Tocilizumab 400mg/Sodium Chloride 100ml/hr IV BID, and Baricitinib 4mg PO BID. Patient has remained in our institution for two months, currently in the ICU in a stable condition slowly being weaned off Fentanyl for eventual removal of mechanical ventilation. Serial chest x-ray show improvement in the lower lungs bilaterally.

\section{Patient 4.}

A 62-year-old female with a PMH of HTN, Parkinson disease, gastroesophageal reflux disease (GERD), schizophrenia, osteoporosis, and vascular dementia presented to ED with syncope and altered mental status. Patient resides in a skilled nursing facility and was found by one of the facility's nurse. She denied any trauma to the head. The nurse stated the patient was experiencing vomiting and fever for a week prior to presentation. She denied associated symptoms such as dyspnea, diarrhea, anosmia, loss of appetite, or cough. SARS-CoV-2 RT-PCR was ordered and she subsequently tested positive and was admitted to the ICU. Patient was treated with Remdesivir $100 \mathrm{mg} /$ Sodium Chloride 250ml/hr IV BID, Hydroxychloroquine Sulfate 200mg PO Q12HR and prophylactically with Heparin Sodium 5,000 units Q12HR SC. AP portable radiograph revealed minimal patchy infiltration in the lung bases bilaterally. Patient was mechanically ventilated for additional 2 weeks and was eventually discharged after marked improvement.

\section{Patient 5.}

An 85-year-old male with a past medical history of CHF, CAD, AFib, CKD, HTN, DM, GERD, and urinary tract obstruction with suprapubic catheter presented to ED from nursing home with a fever and an oxygen saturation of $72 \%$ on CPAP. Patient's CPAP was removed, and he subsequently stopped breathing and went into asystole rhythm. Patient was then resuscitated, intubated, and mechanically ventilated. ROSC was achieved after 5 minutes. Per patient's daughter, he tested positive for COVID-19 three weeks prior to presentation. Although he was asymptomatic, he was isolated and only began experiencing symptoms two days prior to presentation. Patient became dyspneic and his 02 saturation began decreasing causing him to be placed on CPAP. A SARS-CoV-2 RT-PCR confirmed patient's positive COVID-19 status. He was transferred to the ICU and mechanically ventilated with an endotracheal tube. On chest x-ray, patchy areas of infiltrate in the retrocardiac region and right lower lung field with a small effusion were seen. Patient's body temperature continued to slowly rise while chemistry labs demonstrated lactic acidosis indicative of sepsis. His treatment consisted of antibiotics to treat the sepsis, Remdesivir $100 \mathrm{mg} /$ Sodium Chloride $250 \mathrm{ml} / \mathrm{hr}$ IV QD and he was maintained on his home medication of Warfarin 3mg PO QD in addition to Clopidogrel 300mg PO QD as prophylaxis. Patient remained in the ICU for two weeks with weak weaning parameters. Although his sepsis resolved, his pneumonia due to COVID-19 continued to worsen with minimal changes in his serial chest x-rays. The patient was extubated once he passed the weaning parameters but subsequently expired of continuous complications secondary to COVID-19 infection.

\section{Patient 6.}

A 28-year-old female with no past medical history presented to the ED with fever, dry cough and dyspnea for the past two days. Patient reports recent exposure to a patient with an active COVID-19 infection while working as a physician in the inpatient psychiatric unit without proper personal protective equipment (PPE). She reports dyspnea 
that is exacerbated with exertion and slightly improved with rest. However, she denies chest pain, palpitations, hemoptysis, vomiting, changes in bowel habits, and recent travel. History of recent exposure indicated a SARS-CoV-2 RT-PCR which confirmed an active COVID-19 infection. AP portable chest radiograph was normal without any pulmonary infiltration. Patient was subsequently admitted for observation and treated with Acetaminophen 500mg PO BID and Apixaban 2.5mg PO BID as prophylaxis. Marked improvements were noted in the patient's respiration and cough after two days which resulted in discharge with continued prophylaxis of Apixaban for a duration of one month. During a one-week follow-up, patient reported complete resolution of symptoms four days after discharge.

\section{Patient 7.}

A 33-year-old female with no significant past medical history was brought to the emergency department (ED) with complaints of fever, cough, and shortness of breath for 3 days alongside altered mental status and left sided weakness with sensory loss of one-hour duration. Patient reports recent exposure to a roommate with an active COVID-19 infection. There was no significant tobacco, alcohol or illicit drug use history. The patient tested positive via a SARS-CoV-2 RT-PCR test and chest X-ray showed bibasilar opacities. Transthoracic echocardiogram provided normal results. Non-enhanced CT imaging revealed a focal hyper density of the middle cerebral artery (Fig. 6). She was treated with intravenous alteplase, hydroxychloroquine, and azithromycin. Neurological and respiratory symptoms gradually began to improve over the ensuing days. The patient was eventually discharged home on antiplatelet (apixaban ) therapy. During a 1-month follow up, the patient further notes drastic improvement of symptoms.

We have reported on 7 patients with COVID-19 infection in two hospitals. All seven patients were positive for COVID19 by RT-PCR or antibody testing. Six patients (85\%) had positive x-ray findings such as patchy infiltrates, and bilateral lung opacities (Table 1). Four patients of patients received mechanical ventilation. Specific antiviral treatment (remdesivir) was given to 4 patients. One patient received clopidogrel and warfarin. Five patients received hydroxychloroquine. Patients were administered a variety of anticoagulation therapy as seen in table 2. Most of the patients survived (85\%) and one died. The one mortality had many co-morbid conditions including; $\mathrm{CHF}$, CAD, AFib, CKD, HTN, and DM.

\begin{tabular}{|c|c|c|c|c|c|c|c|}
\hline $\begin{array}{l}\text { Radiological } \\
\text { investigations }\end{array}$ & Patient 1 & Patient 2 & Patient 3 & Patient 4 & Patient 5 & $\begin{array}{l}\text { Patient } \\
6\end{array}$ & Patient 7 \\
\hline X-ray finding & $\begin{array}{l}\text { bilateral } \\
\text { lung } \\
\text { opacities }\end{array}$ & $\begin{array}{l}\text { patchy } \\
\text { peripheral } \\
\text { infiltrates in } \\
\text { the right } \\
\text { midlung } \\
\text { laterally } \\
\text { and smaller } \\
\text { patchy } \\
\text { peripheral } \\
\text { infiltrates in } \\
\text { the left } \\
\text { midlung } \\
\text { respectively }\end{array}$ & $\begin{array}{l}\text { diffuse } \\
\text { airspace } \\
\text { consolidation } \\
\text { involving the } \\
\text { right lung, } \\
\text { retrocardiac, } \\
\text { and left base } \\
\text { suspicious } \\
\text { for } \\
\text { pneumonic } \\
\text { airspace } \\
\text { infiltrate }\end{array}$ & $\begin{array}{l}\text { minimal } \\
\text { patchy } \\
\text { infiltration } \\
\text { in the } \\
\text { lung } \\
\text { bases } \\
\text { bilaterally }\end{array}$ & $\begin{array}{l}\text { patchy } \\
\text { areas of } \\
\text { infiltrate in } \\
\text { the } \\
\text { retrocardiac } \\
\text { region and } \\
\text { right lower } \\
\text { lung field } \\
\text { with a } \\
\text { small } \\
\text { effusion } \\
\text { were seen }\end{array}$ & Normal & $\begin{array}{l}\text { bibasilar } \\
\text { opacities }\end{array}$ \\
\hline
\end{tabular}

Table 1: Radiological Data 


\begin{tabular}{|llllllll|}
\hline $\begin{array}{l}\text { Management and } \\
\text { outcome }\end{array}$ & Patient 1 & Patient 2 & Patient 3 & Patient 4 & Patient & Patient 6 & Patient 7 \\
\hline Ventilatory support & - & + & + & + & + & - & - \\
Clopidogrel & - & - & - & - & + & - & - \\
\hline Warfarin & - & - & - & - & + & - & - \\
$\begin{array}{l}\text { Hydroxychloroquine } \\
\text { Sulfate }\end{array}$ & + & + & + & + & - & - & + \\
Antiviral (Remdesivir) & - & + & + & + & + & - & - \\
\hline Apixaban & + & + & - & - & - & + & + \\
\hline Heparin & - & + & - & + & - & - & - \\
\hline Enoxaparin & - & + & + & - & - & - & - \\
\hline Tocilizumab & - & - & + & - & - & - & - \\
\hline Baricitinib & - & - & + & - & - & - & - \\
\hline Alteplase & - & - & - & - & - & - & + \\
\hline Antibiotics & - & - & - & - & - & - & + \\
\hline Outcome & Recovery & Recovery & Recovery & Recovery & Death & Recovery & Recovery \\
\hline
\end{tabular}

Table 2: Management and Outcome

\section{Discussion}

The novelty of COVID-19 is slowly fading as more studies are exploring the etiologies of this deadly virus. It is well known now that the mortality of this pathogen is rooted in hypoxemia due to the initiation and exacerbation of acute respiratory distress syndrome (ARDS). However, increasing amount of studies are suggesting an accompanying contribution of thromboembolic events [5,6]. Also, venous thromboembolism has been demonstrated on both microvascular and macrovascular scales in published post-partum studies in COVID-19 patients [7]. The pathophysiology of this is still very much under investigation but various hypotheses have touched upon the possibility of the immuno-thrombotic phenomeno [1]. McGonagle et al. suggests that the binding of SARS-CoV-2 to the angiotensin-converting enzyme 2 (ACE2) ultimately leads to a generalized pulmonary hypercoagulable state due to activation of type II pneumocytes propagating an inflammatory cascade $[8,9]$. The subsequent cytokine storm leads to diffuse alveolar damage and immunostimulatory molecules invading microvasculature, thus leading to the phenomenon of immuno-thrombosis. A novel hypothesis by Hong-Long Ji et al. suggested that plasmin might be responsible for cleaving the furin sites of spike "S" proteins of SARS-CoV-2, which would then increase the infectivity of respiratory cells by the virus [10]. This would suggest, in theory, that disease severity can be measured via plasmin, plasminogen, and D-dimer levels, thus opening the possibility of utilizing antiplasmin compounds as a potential mode of therapy [10]. 
Moreover, the case reports above highlight some of the pathological findings that might arise as a result of a direct COVID-19 infection. Based on the reported findings, it is appropriate to suggest that there is a direct or an indirect correlation between COVID-19 and the development of coagulopathies. Next, one of our seven patients suffered an ischemic brain stroke and developed a prothrombotic state secondary to COVID-19 infection. Whereas patients most placed on prophylactic thrombolytics achieved a positive outcome with reduced risk of thromboembolic complications. Although there are limited data available to inform clinical management surrounding prophylactic use of thrombolytics in COVID-19 patients, many hospitals began implementing it, due to the increased risk for venous and arterial thrombosis of large and small vessels [11]. Therefore, understanding the pathophysiology, the coagulation cascade is a critical and fundamental part of appropriate therapeutic development and management for patients with COVID-19. The coagulation cascade is the physiological activation of a series of clotting factors that leads to secondary hemostasis, a physiological process that rapidly prevents and stops spontaneous bleeding at the site of injury. This pathway comprises of two independent paths, intrinsic and extrinsic, that converge at a common point known as the common pathway, which serves to ultimately convert fibrinogen to fibrin, a necessary clotting factor to stabilize the platelet plug formed during primary hemostasis in order stop bleeding.

In addition to the coagulation promoting factors that exist in the blood stream, there are also inhibitory substances which downregulate coagulation. For example, Protein $\mathrm{C}$ and $\mathrm{S}$ act to prevent coagulation, mainly by inactivating factors V and VIII, respectively. Moreover, thrombin (factor II), a procoagulant serves as a negative feedback by activating plasminogen to plasmin and stimulating the production of antithrombin (AT). Plasmin acts directly on the fibrin mesh and breaks it down. AT decreases the production of thrombin from prothrombin and decreases the amount of activated factor $\mathrm{X}$.

Normal hemostasis strikes a balance between the pro-coagulant system (platelets and coagulation cascade) that is responsible for clot formation and the anticoagulant system, responsible for breaking down clots. If hemostatic dysregulation occurs due to a defect in one of these systems, then either thrombosis or excessive bleeding may occur especially in the perioperative or during critical illness period. Therefore, a balance between clot formation and fibrinolysis activation are essential to limiting detrimental extension of vessel clotting.

Many varieties of microorganisms have been shown to directly activate the blood coagulation system and component of the blood hemostatic systems involved in the immune response and immune system modulations. It has been hypothesized that the direct activation of coagulation is a beneficial response that aids in limiting pathogen dissemination, augmenting innate and adaptive immunity, and repair of damaged tissues. Generally, components of viral pathogens directly activate the coagulation cascade either by increased levels of clotting factors (e.g., factor VIII, factor XI), soluble tissue factor, and von Willebrand factor or a decrease levels of the anticoagulants, protein $\mathrm{C}$, protein $\mathrm{S}$, antithrombin, and tissue factor pathway inhibitor. Other markers that may indicate activation include increased thrombin generation, platelet activation, fibrin degradation and fibrinolysis (e.g., D-dimer and plasmin-a2-antiplasmin complexes). Although the main function of thrombin is to promote clot formation by activating platelets and by converting fibrinogen to fibrin, thrombin also exerts multiple cellular effects and can further augment inflammation via proteinase-activated receptors (PARs), principally PAR-1, the main thrombin receptor that mediates thrombin-induced platelet aggregation as well as the interplay between coagulation, inflammatory, and fibrotic responses. All of which are important aspects of the pathophysiology of fibroproliferative lung disease, such as seen in COVID-19. 
Viral infections likely drove evolution to activate this coagulation cascade as a host defense mechanism in order to limit spread of the pathogen. Unfortunately, acute viral infections can also overstimulate this cascade during an immune response leading to multiple negative consequences such as micro-thrombosis, disseminated intravascular coagulation (DIC), subsequent hemorrhage, and ultimately multiorgan failure and mortality- evidenced in severe COVID-19 pneumonia with raised d-dimer concentrations being a poor prognostic feature and DIC being commonplace in non-survivors.

Tissue factor (TF) plays a major role in the activation of the coagulation cascade during viral infection by several different mechanisms as observed in different viruses. Firstly, TF expression is increased in endothelial cells infected with human simplex virus (HSV) and Dengue virus [12]. Secondly, Ebola virus infection induces TF expression in lymphoid macrophages and circulating blood cells, which is associated with Ebola associated hemorrhagic fever [13]. Thirdly, the toll like receptor 3 (TLR3) - the host's apparatus for recognizing viral DNA, induces TF expression in cultured endothelial cells and activates the coagulation system in mice. In a rhesus monkey model of Ebola virus hemorrhagic fever, direct inhibition TF expression was observed to reduce the cytokine storm and mortality - further evidence that TF plays such a pivotal role in viral activation of the coagulation cascade [14].

Following the inflammatory immuno-thrombosis model, Rico-Mesa et al. suggests the use of heparin not only as an anticoagulant but concurrently as an anti-inflammatory as well. This two-pronged approach shows promise in terms of halting the reciprocal relationship between coagulation and the immune system [15]. Reducing thrombin generation, in theory, should lessen the intensity of the inflammatory response to COVID-19, and subsequently the resulting ARDS. A recent meta-analysis displayed that supplementing disease management with LMWH within 7 days of initial onset of the disease decreased the risk of 7-day mortality by 48\% [16]. By no means does this mean the end of COVID-19 but such studies and tested hypotheses seem to be pointing us in the right direction.

Furthermore, anticoagulation therapy has been used in our institutions due to the increased risk of thromboembolic events. Anticoagulation therapy is commonly prescribed to patients with conditions such as atrial fibrillation, deep vein thrombosis (DVT), hip or knee replacement surgery, ischemic stroke, myocardial infarction (MI), pulmonary embolism (PE), and unstable angina in an attempt to decrease the risk of thromboembolic events [7].

Anticoagulation medications are also given to patients with extended hospitalization to prevent the risk of DVT and its concomitant progression to PE. Therapy is based on the comorbid condition and is targeted to prevent the progression of disease. However, the therapy is not without inherent risks, and each medication is unique in its adverse effects. Anticoagulation medications are divided into classes based on the target and mechanism of action [17].

There are 4 classes of anticoagulants:

1. The indirect thrombin and Factor XA inhibitors (Heparins \& Heparinoids)

2. Vitamin $\mathrm{K}$ antagonists (Coumadin, 1,3 indandiones, and Tioclomarol)

3. Direct thrombin inhibitors (Univalent \& Bivalent)

4. Direct factor XA inhibitors

Indirect thrombin and factor XA inhibitors 
These medications include unfractionated heparin (Fragmin), low-molecular-weight heparins (Lovenox), and Fondaparinux (Arixtra). Heparin has a distinct mechanism of action where antithrombin III inhibits clotting factor proteases such as thrombin, factors IXA and XA, but it does so in a relatively slow manner. Heparin acts as a cofactor for the antithrombin-protease reaction where it binds to antithrombin III and accelerates the inhibition of those factors. Heparin is an injectable, rapidly acting anticoagulant, used to interfere with the formation of thrombi. The unfractionated and low molecular weight heparin $(\mathrm{LMWH})$ are a mixture of straight chain sulfated mucopolysaccharides which are normally isolated from bovine lung or porcine intestinal mucosa. LMWH inhibits factor XA with high affınity but inhibits thrombin with a lesser affınity, while unfractionated heparin (UFH) inactivates thrombin and factor XA with the same affinity. LMWH has equal efficacy to UFH but has higher bioavailability with a longer half-life and less frequent dosing which is a common reason to replace UFH in clinical practice. Heparin levels can be monitored by performing the activated partial thromboplastin time (aPTT) assay which is a test for the intrinsic and common pathways of coagulation. When a patient is administered LMWH, the dosing is predictable in plasma levels and it is not necessary to measure LMWH blood levels. Heparin is used in DVT, PE, MI, and is the drug of choice during pregnancy. Adverse reactions include bleeding, hypersensitivity reactions, and the most dangerous being Heparin-induced thrombocytopenia (HIT). The risk of HIT is lower with LMWH than with heparin and minimal with fondaparinux4. Excessive anticoagulation is treated by discontinuing heparin, if bleeding occurs, protamine sulfate is administered.

Heparin Induced Thrombocytopenia (HIT)

When a patient is administered heparin, antibodies can recognize various complexes of heparin and platelet factor 4 (PF4) leading to the formation of a complex between the two. IgG binds to heparin/PF4 and forms an immune complex which can bind to the FC receptor on the surface of platelets leading to their activation, degranulation and aggregation [11]. The activated platelets release more PF4 which can bind more heparin and lead to a vicious cycle causing thrombocytopenia and thrombosis. The treatment of choice for HIT is to discontinue heparin and administer a direct thrombin inhibitor such as argatroban or fondaparinux.

Vitamin K Antagonists

Vitamin $\mathrm{K}$ is involved in the coagulation cascade and it activates factors II, VII, IX, X, protein C, and protein S. Vitamin $\mathrm{K}$ antagonists such as warfarin (Coumadin) inhibit vitamin $\mathrm{K}$ epoxide reductase inhibiting the function of vitamin $\mathrm{K}$, leading to inactivation of the clotting factors due to the lack of $Y$-carboxyglutamyl side chains. Anticoagulation with warfarin takes several hours as the coagulation factors involved have half-life's ranging from 6- 60 hours, thus the anticoagulation effect becoming apparent within 24 hours of warfarin administration and peaking within 72 - 96 hours. In reciprocation, this also means that reversal of warfarin takes approximately 24 hours to become apparent. Warfarin also has a narrow therapeutic index, and it can have many drug-drug interactions so it must be regularly monitored. Monitoring warfarin is done by measuring the prothrombin time (PT) which tests the function of the extrinsic and common pathways in the coagulation cascade. Warfarin is used in the prevention and treatment of DVT and PE following an initial dose of heparin. Adverse effects of warfarin include hemorrhage and necrosis of the 
skin due to reduced activity of protein $\mathrm{C}$. It is also contraindicated during pregnancy because it can cross the placenta and lead to hemorrhagic disorders and other birth defects in the fetus [11].

Direct thrombin inhibitors

Direct thrombin Inhibitors (DTIs) are a class of anticoagulants that directly inhibit thrombin and prolong clotting. They are mainly used in HIT and acute coronary syndrome. DTIs are classified as either univalent or bivalent according to the binding site on thrombin [7] The bivalent DTIs include bivalirudin (Angiomax), desirudin (Iprivask), and lepirudin (Refludan). They bind to the active as well as the exo-site 1 of thrombin. The Univalent medications include argatroban, dabigatran (Pradaxa), delagatran (Exanta), and ximelagatran (Exarta), and they only bind to the active site of thrombin. Bivalirudin is enzymatically eliminated and is safest to use in the presence of both hepatic and renal dysfunction [7]. Lepirudin is metabolized mainly by the kidneys and the dose is adjusted in patients with renal dysfunction [7]. Argatroban is mainly metabolized by the liver so it is not recommended in patients with hepatic dysfunction. It is used during percutaneous coronary intervention (PCl) in patients with or at risk of HIT [7]. Dabigatran (also called dabigatran etexilate) is absorbed and converted to an active metabolite in the liver, it is used in the prevention of stroke in patients with non-valvular atrial fibrillation and routine laboratory monitoring is not recommended [7]. Monitoring the therapeutic efficacy of DTIs is done via measuring aPTT but it is nonspecific as those agents are relatively new and there has been an increased focus on developing laboratory tests to measure DTI levels in the blood [7].

Direct factor XA inhibitors

Direct factor XA inhibitors include apixaban (Eliquis) and rivaroxaban (Xarelto). They are oral medications that have similar antithrombotic efficacy to warfarin and lower bleeding rates. They have a rapid onset of action, wider therapeutic window, fewer drug-drug interactions, and no monitoring requirements making them an ideal alternative to warfarin when used in the prevention and treatment of venous thromboembolism as well as pulmonary embolism.

\section{Conclusions}

As this case series of 7 patients demonstrates, COVID-19 infection can cause disruption of physiological and pathophysiological homeostasis, leading to abnormal coagulation parameters. The diagnosis of COVID-19 was confirmed by utilizing PT-PCR and imaging studies. Additionally, abnormal brain computed tomography scan of the brain validated the diagnosis of ischemic brain changes secondary to COVID-19 infection. Unlike most coronavirus infections that mainly cause pulmonary symptoms, this case of coronavirus infection led to profound hemostatic dysregulation. Future prospective registries to study this correlation, as well as studies of anticoagulation therapy to prevent these potentially life-threatening events are underway. We believe that patients who present with stroke related symptoms during the pandemic, a thoroughly investigation of COVID-19 infection should be initiated, and anticoagulation therapy should be considered. Conversely, a neurological examination should be done repeatedly throughout the course of illness, to screen for focal neurologic deficits, altered consciousness, confusion, seizure, and other related stroke symptoms.

\section{Declarations}


The authors received verbal consent from all participants in this study and Richmond University Medical Center Ethics Committee. Furthermore, they did not utilize any animal subjects or tissue in this study.

In compliance with the ICMJE uniform disclosure form, all authors declare the following:

Payment/services info: All authors have declared that no financial support was received from any organization for the submitted work.

Financial relationships: All authors have declared that they have no financial relationships at present or within the previous three years with any organizations that might have an interest in the submitted work.

Other relationships: All authors have declared that there are no other relationships or activities that could appear to have influenced the submitted work.

\section{References}

1. Ni, W., Yang, X., Yang, D: Role of angiotensin-converting enzyme 2 (ACE2) in COVID-19. Crit Care 24. 422

2. Prieto-Pérez, L., Fortes, J., Soto, C: Histiocytic hyperplasia with hemophagocytosis and acute alveolar damage in COVID-19 infection. Mod Pathol. 10.1038/s41379-020-0613-1

3. Becker R: C: ( 2020: COVID-19 update: Covid-19-associated coagulopathy. Journal of thrombosis and thrombolysis. 50:54-67. 10.1007/s11239-020-02134-3

4. Furnish, T., \& Wallace, M: S: 2014: Anticoagulant Therapy. Retrieved September 30. 2020, from https://www.sciencedirect.com/topics/medicine-and-dentistry/anticoagulant-therapy, 5:101016. 10.1016/B978-1-4557-0101-8.00007-2

5. Ellul, MA. Benjamin, L. Singh B: Neurological associations of COVID-19. Lancet Neurol. 2020, 19:767783. 10.1016/S1474-4422(20)30221-0

6. Cui, S, Chen, S, Li, X: Prevalence of venous thromboembolism in patients with severe novel coronavirus pneumonia. J Thromb Haemost. 2020, 18:1424. 10.1111/jth.14830

7. Rhea, J. M. (2011, August 1: Please Enable Cookies. Retrieved, Accessed. Accessed: September 30, 2020, from: https://www.mlo-online.com/home/article/ 13004081/direct-thrombin-inhibitors-clinical-uses-mechanism-ofaction-and-lab.

8. Rico-Mesa, J.S., White, A. \& Anderson, A.S: Outcomes in Patients with COVID-19 Infection Taking ACEI/ARB. Curr Cardiol Rep. 22. 31:10.1007/s11886-020-01291-4

9. Hoffmann, M., Kleine-Weber, H., Schroeder, S: SARS-CoV- 2. Cell Entry Depends on ACE2 and TMPRSS2 and Is Blocked by a Clinically Proven Protease Inhibitor. Cell. 181:271-280. 10.1016/j.cell.2020.02.052

10. Ji, H., Zhao, R., Matalon, S: Elevated Plasmin(ogen) as a Common Risk Factor for COVID-19 Susceptibility. Physiological Reviews. 100:1065-1075. 10.1152/physrev.00013.2020

11. Tang N, Li D, Wang X, Sun Z: Abnormal coagulation parameters are associated with poor prognosis in patients with novel coronavirus pneumonia. J Thromb Haemost Feb. 2020, 10.1111/jth.14768

12. Ginsberg, J., Crowther: M., White: R: January. 01). Anticoagulation Therapy. Retrieved, Accessed, Accessed, Accessed: September 30, 2020,

from: https://ashpublications.org/hematology/article/2001/1/339/18621/Anticoagulation-Therapy...

13. Palta, Sanjeev: Overview of the coagulation system. Indian journal of anaesthesia vol. 58,5. 2014, 51523. $10.4103 / 0019-5049.144643$

Page $11 / 18$ 
14. Morales-Vidal, S., Schneck: M., Flaster: Classification of anticoagulant drugs. Examples of specific... Retrieved, Accessed: September 30. 2020, from: https://www.researchgate.net/figure/Classification-of-anticoagulantdrugs-Examples-of-specific-drugs-are-cited-Note-t. 10.1586/ern.11.185

15. Wichmann: Dominic: Autopsy Findings and Venous Thromboembolism in Patients With COVID- 19: A Prospective Cohort Study. Annals of internal medicine vol. 173,4. 2020, 268:277. 10.7326/M20-2003

16. Rico-Mesa, J., Rosas, D., Ahmadian-Tehrani, A: The Role of Anticoagulation in COVID-19-Induced Hypercoagulability. Current Cardiology Reports. 22. 7:10.1007/s11886-020-01328-8

17. Li J, Li Y, Yang B, Wang H, Li L: Low-molecular-weight heparin treatment for acute lung injury/acute respiratory distress syndrome: a meta-analysis of randomized controlled trials. Int J Clin ExpMed. 2018, 11:414-22. 11:41422

\section{Figures}

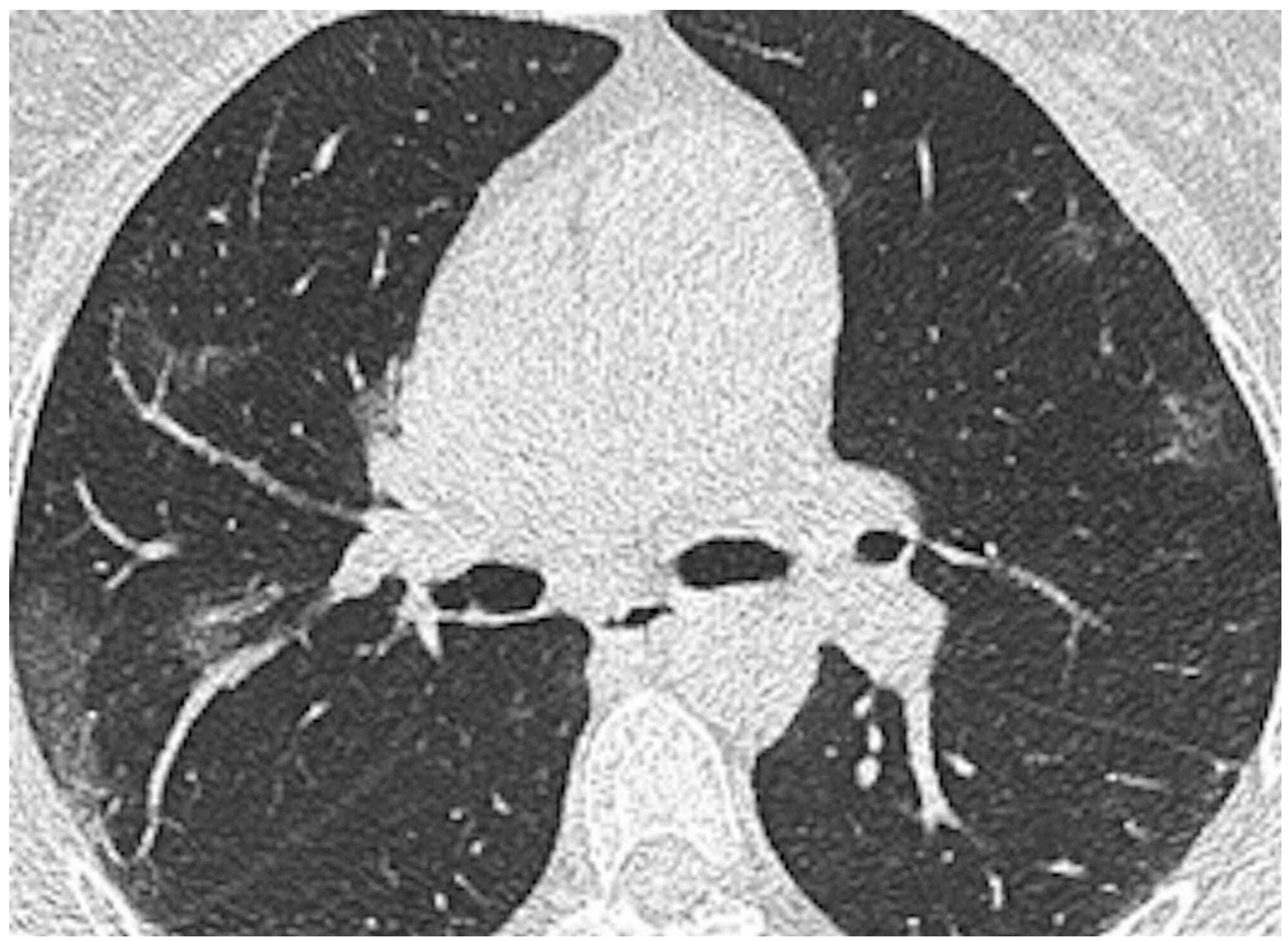

\section{Figure 1}

Unenhanced CT image of the chest in an 88-year-old reflecting mild form of COVID-19 infection. CT scan demonstrating ground-glass opacities in both upper lobes. 

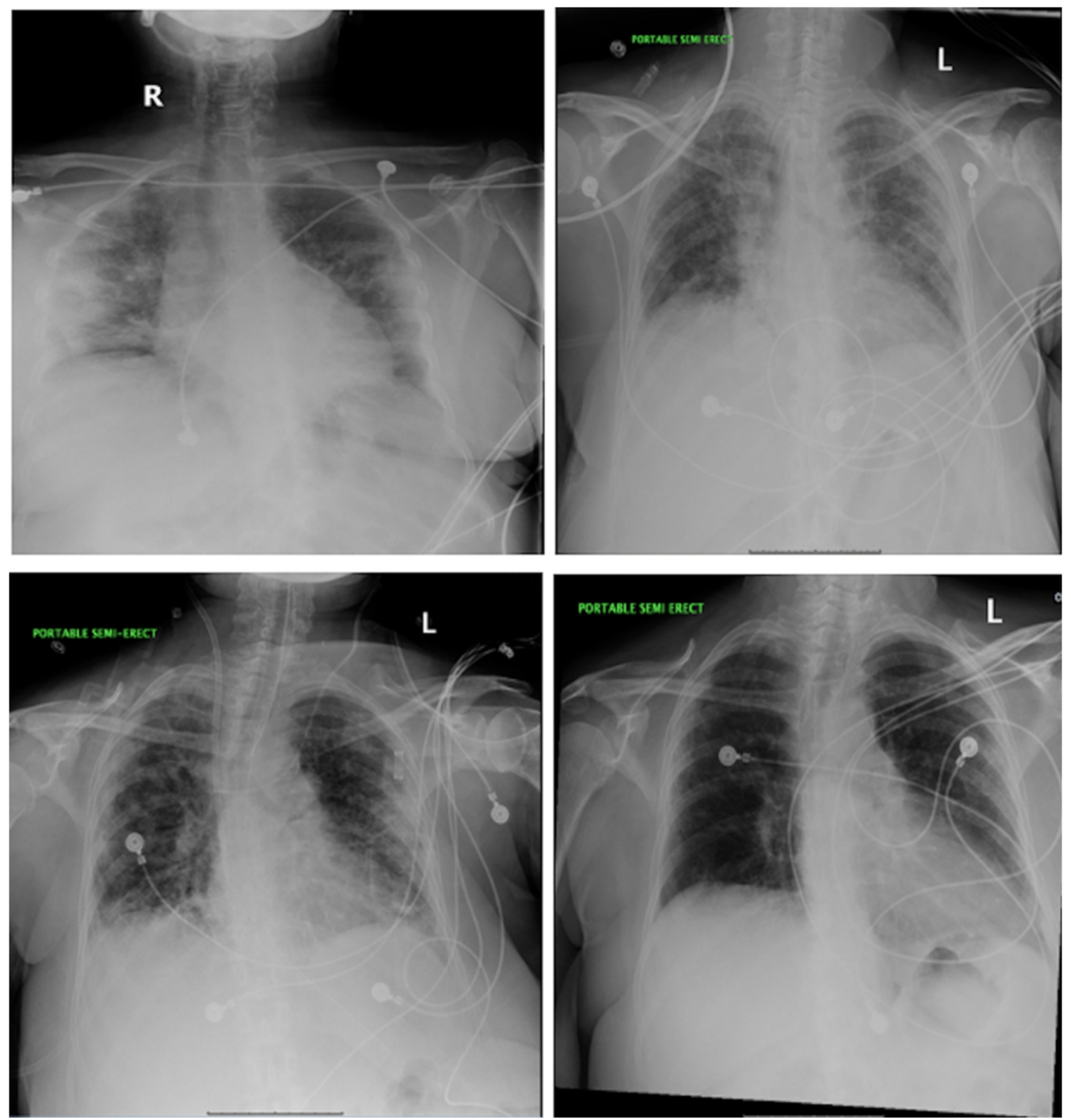

\section{Figure 2}

Serial chest x-rays of a 64-year-old female demonstrating patchy infiltration, predominantly in the midlungs bilaterally, left pleural effusion and slight atelectasis, leading into start of resolution. 

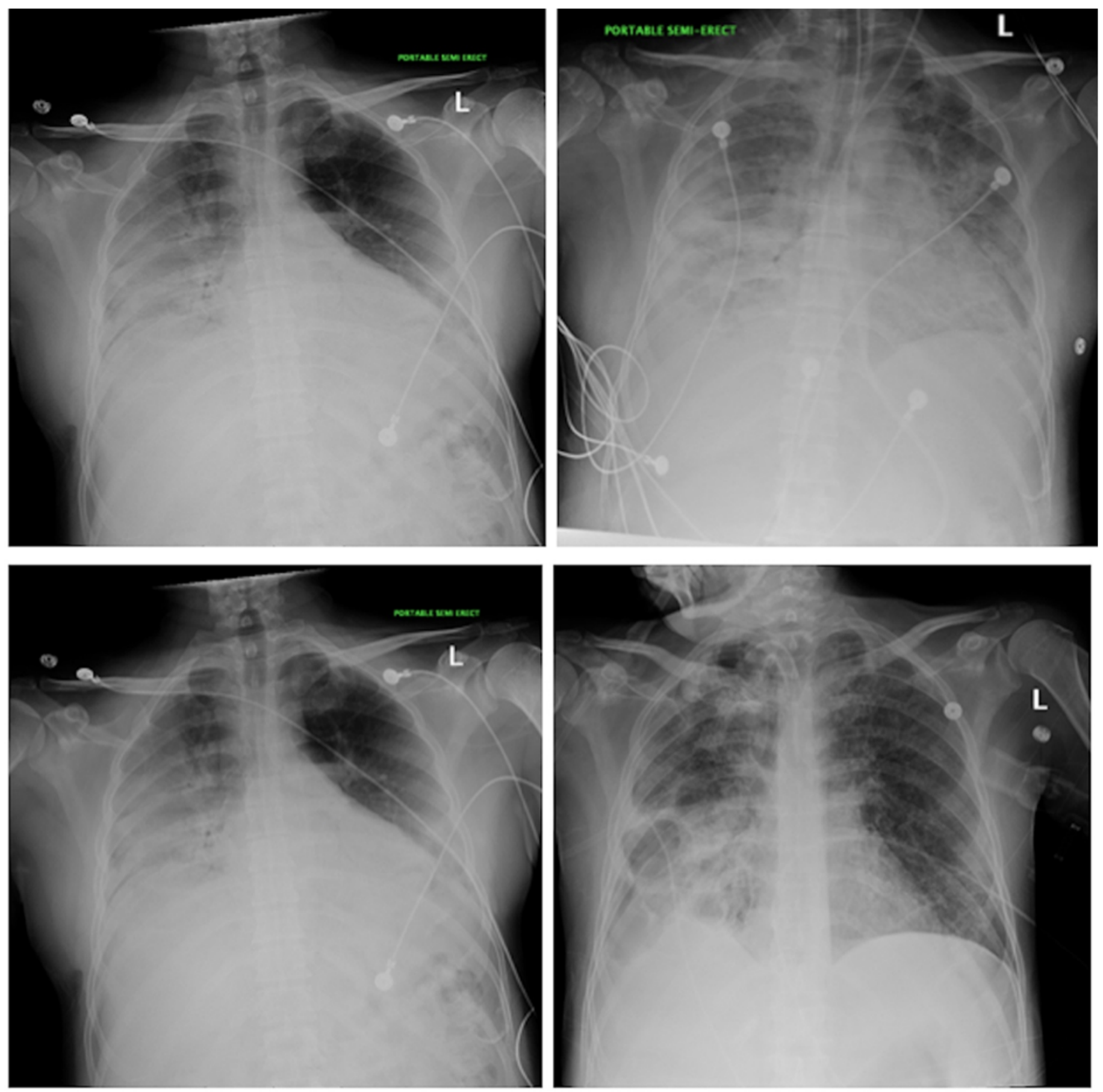

\section{Figure 3}

Serial chest X-rays showing diffuse airspace consolidation involving the right lung, retrocardiac area and left base, suspicious for airspace infiltrates indicative of COVID pneumonia. 

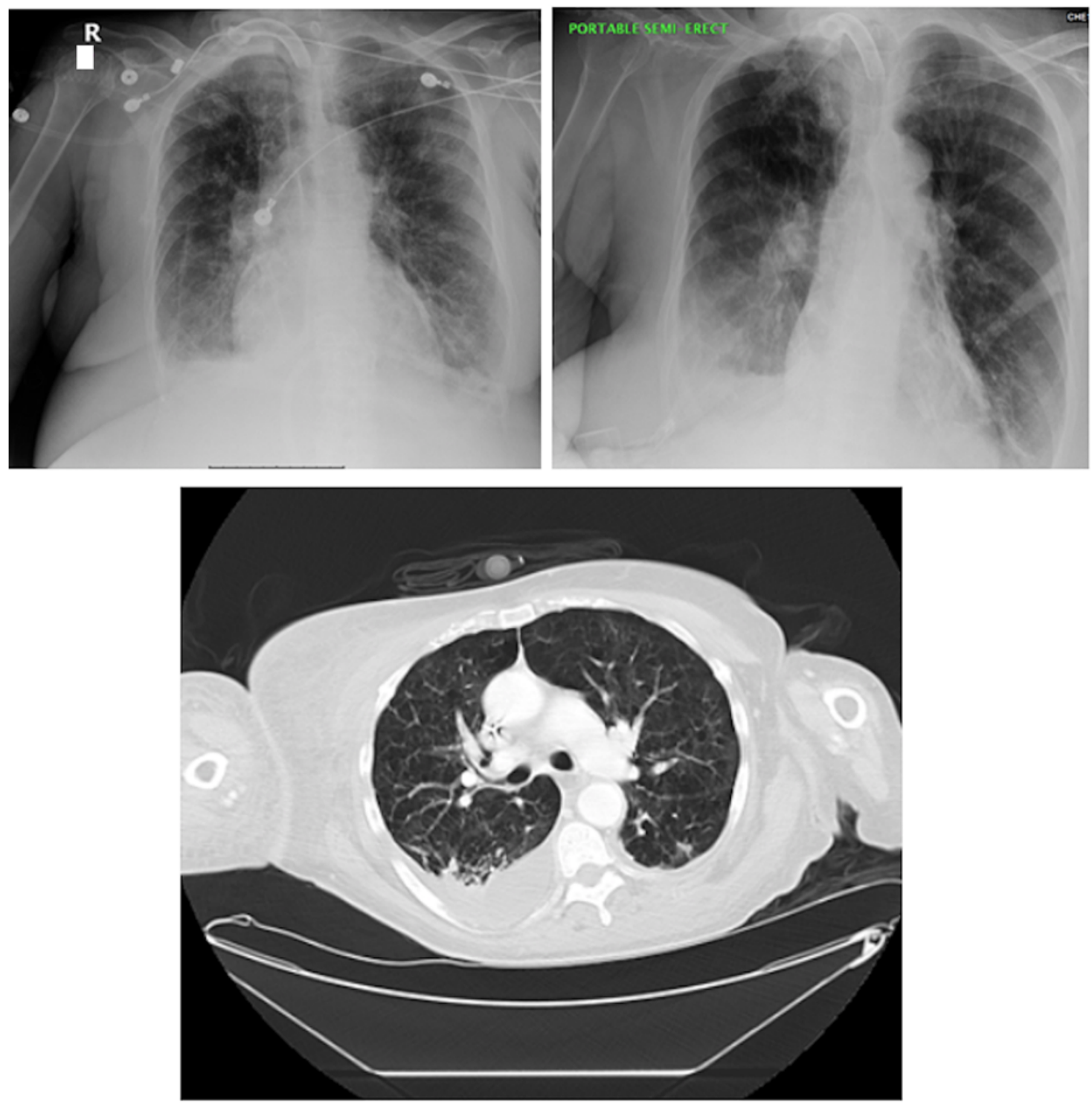

Figure 4

Two serial chest x-rays demonstrate clear patchy infiltration. Ground glass opacities visible on CT of chest. 

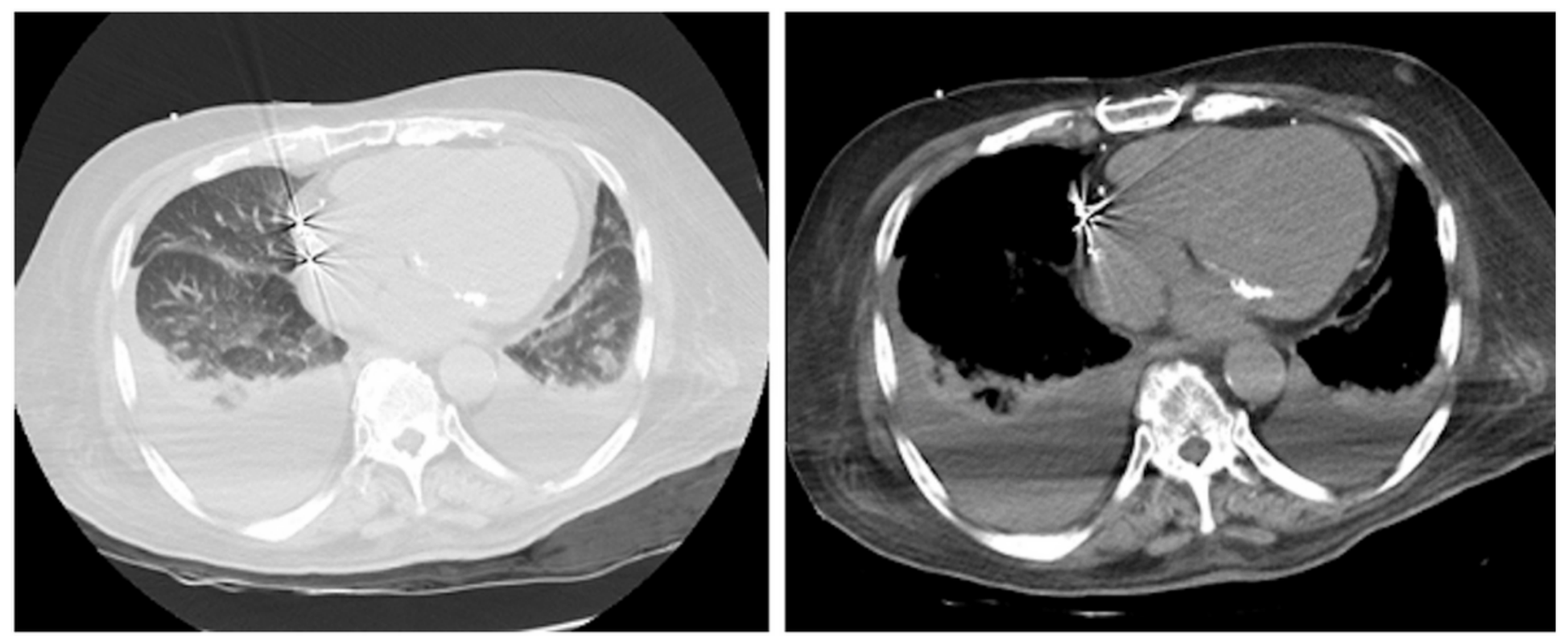

Figure 5

CT of chest showing bilateral base atelectasis and patchy infiltration. 


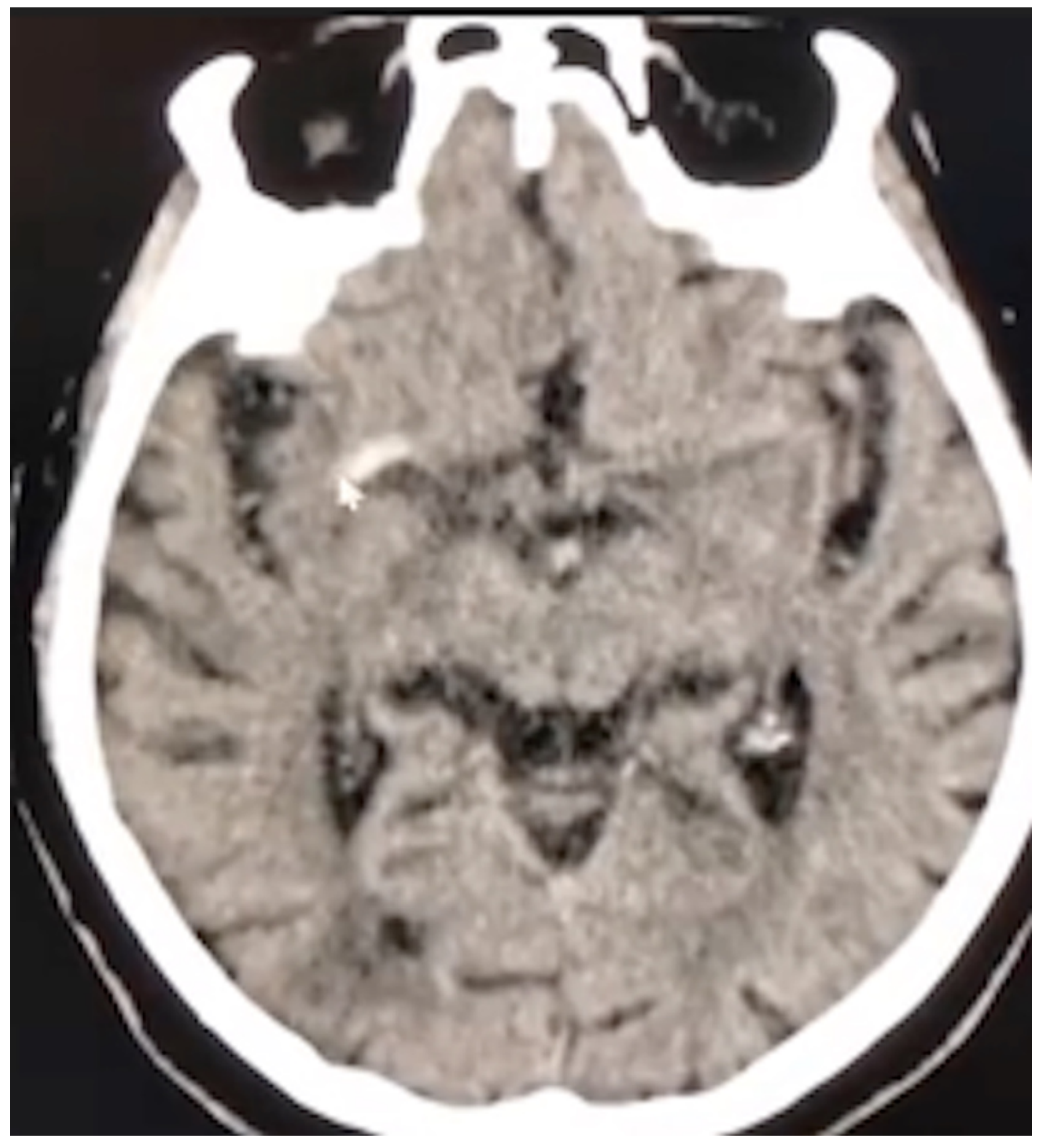

Figure 6

Non-enhanced CT image showing hyperdense MCA sign. 


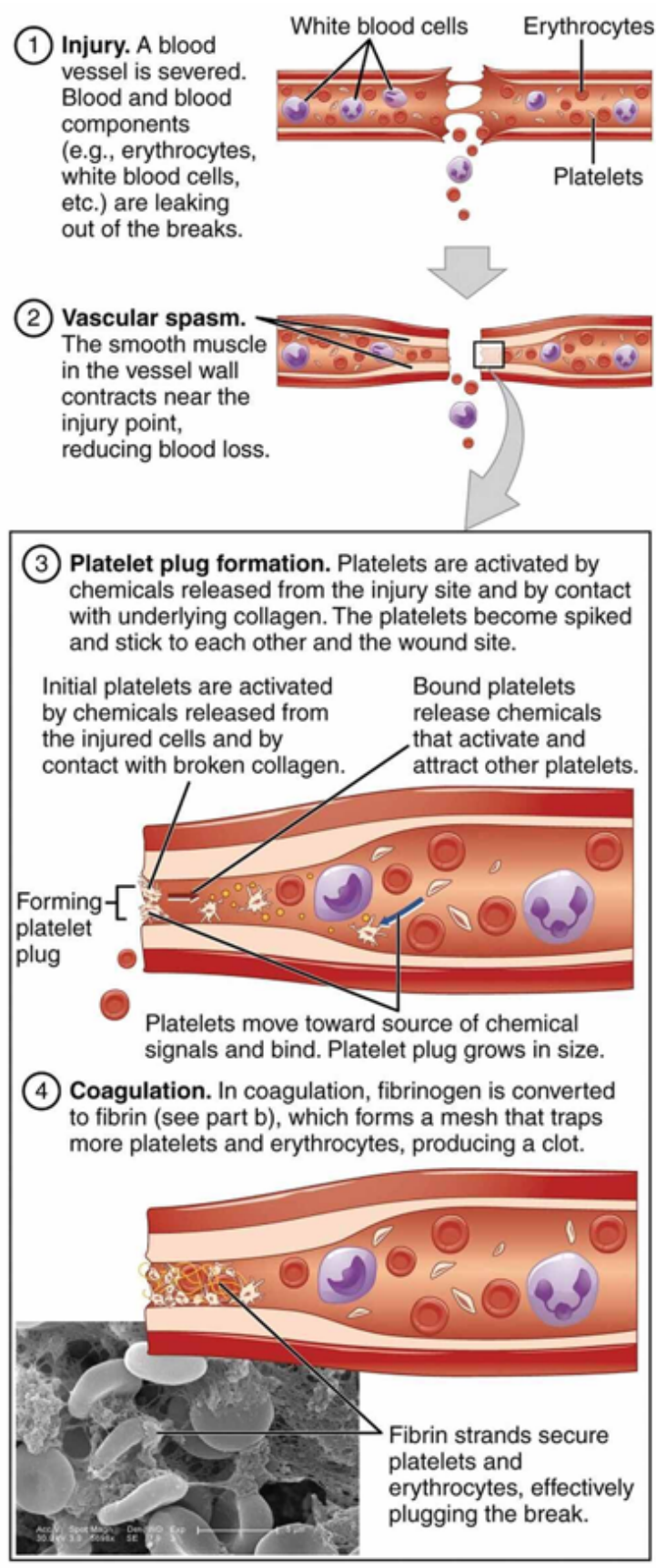

(a) The general steps of clotting

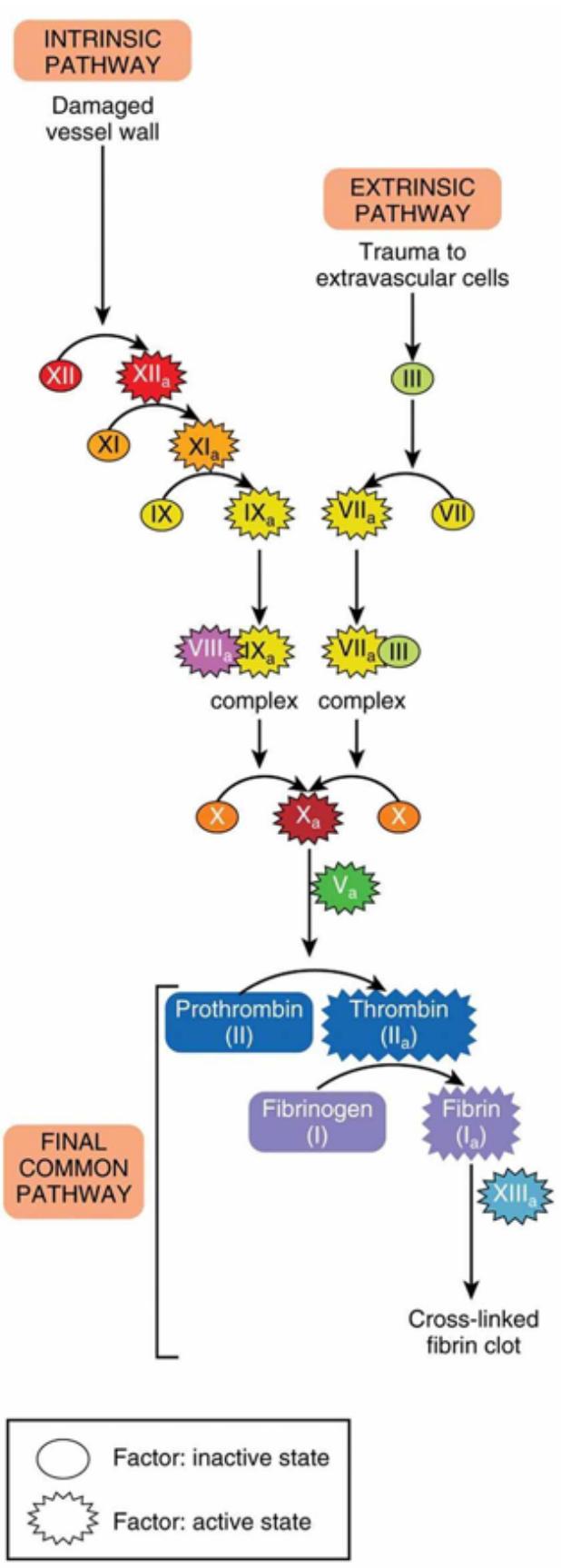

(b) Fibrin synthesis cascade

\section{Figure 7}

(a) A targeted insult to a blood vessel causes an activation of the process of hemostasis. Blood clotting involves three significant steps. Initially, vascular spasm will result in constriction flow of blood. Moreover, a platelet plugs will be formed in order to seal small openings in the vessel. Coagulation will then enable the repair of the vessel wall once the leakage of blood has ended. (b) The synthesis of fibrin in blood clots involves either an intrinsic pathway or an extrinsic pathway, both of which lead to a common pathway. (credit a: Kevin MacKenzie) Credit site: https://courses.lumenlearning.com/ap2/chapter/hemostasis/ 